\title{
Rigid-Vehicle-Loaded Bridge Modal Analysis Using Perturbation Method
}

\author{
Yangmei Cao ${ }^{1,}$, , Zhi Sun ${ }^{2, b}$ \\ ${ }^{1}$ Department of Bridge Engineering, Tongji University, Shanghai, china \\ ${ }^{2}$ State Key Laboratory for Disaster Reduction in Civil Engineering, Shanghai, China \\ acaoym1992@163.com, bsunzhi1@tongji.edu.cn
}

Keywords: modal analysis, perturbation method, mass-beam system.

Abstract. In this paper, modal analysis of rigid-vehicle-loaded girder bridge vibration based on perturbation method is presented. The governing differential equation for the girder bridge free vibration analysis is established by introducing a dimensionless parameter, the relative mass, which is the ratio between the masses of the rigid vehicle and girder bridge. The differential equations at the zero-th and the first order scales are expressed and the first order perturbation terms of the natural frequencies and mode shapes are solved. Case studies on a clamped-clamped girder bridge verify the accuracy of the derivation. The results show that the inertial effect of the rigid-vehicle will induce the maximum variation when the vehicle is located at the peak points of the mode shapes and no variation if the vehicle is located at the inflection points.

\section{Introduction}

The vehicle-bridge coupled vibration problem is a continuous research focus in the related academic area. The variation of the modal parameters of the bridge due to the moving vehicle at different locations is an important topic to understand and analyze the behavior of the coupled system. Different analytic methods are employed to analyze the free vibration characteristics of the idealized mass-beam system [1-3]. Recent works based on perturbation method presented a different idea to conduct the modal analysis of beam structures considering small perturbations due to shear deformation and rotational inertia or crack $[4,5]$. In this study, by modeling the inertial effect of the rigid vehicle as a small perturbation parameter of the bridge structure, the perturbation method is employed to analyze the modal parameters of the idealized mass-beam system.

Based on this idea, the governing differential equation for the bridge free vibration analysis is established with a dimensionless perturbation parameter, the relative mass. The differential equations at the zero-th and the first order scales are expressed and the first order perturbation terms of the natural frequencies and mode shapes are derived. Case studies on a clamped-clamped girder bridge are conduced to verify the method and present some observations.

\section{Theoretical Method}

For a bridge carrying vehicles (as shown in Fig. 1), the mass of the unit length can be expressed as:

$$
m_{d}(x)=m_{0}\left(1+\sum_{i=1}^{m} \varepsilon_{i} L \delta\left(x-x_{d i}\right)\right)
$$

where $m_{0}=\rho A, \varepsilon_{i}=m_{i} / M ; \rho, A$, and $L$ are the density, cross-section area, and length of the bridge, $m_{i}$ and $x_{d i}$ are the mass and the location coordinates of the $i$-th rigid vehicle.

The governing equation for the eigen-value analysis of the system is:

$$
E I \tilde{\phi}_{p}^{\prime \prime \prime \prime}-\tilde{\lambda}_{p} m_{0}\left(1+\sum_{i=1}^{m} \varepsilon_{i} L \delta\left(x-x_{d i}\right)\right) \tilde{\phi}_{p}=0
$$


where $E$ and $I$ are the elastic modulus and the moment of inertia of the girder bridge, $\lambda_{p}^{\%}$ and $\phi_{p}^{\%}$ are the eigen-value and mode shape function of the coupled vibration system.

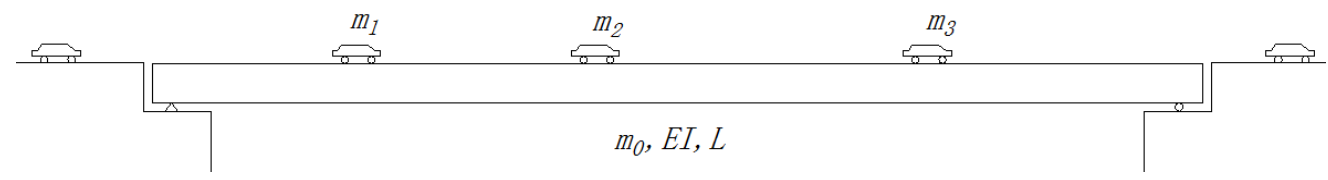

Fig1. A rigid-vehicles-loaded bridge structure

According to the perturbation theory, the modal characteristic parameters of rigid-vehicle-loaded girder bridge can be expressed as:

$$
\left\{\begin{array}{l}
\lambda_{p}^{\%}=\lambda_{p}^{0}+\sum_{i=1}^{m} \varepsilon_{i} \lambda_{p i}^{1}+O\left(\varepsilon^{2}\right) \\
\phi_{p}^{\%}=\phi_{p}^{0}+\sum_{i=1}^{m} \varepsilon_{i} \phi_{p i}^{1}+O\left(\varepsilon^{2}\right)
\end{array}\right.
$$

where $\lambda_{p}^{0}$ and $\phi_{p}^{0}$ is eigen-values and mode shapes of girder bridges, $\lambda_{p}^{0}=k_{p}^{4} \cdot\left(E I_{0} / m_{0}\right) ; \lambda_{p i}^{1}, \phi_{p i}^{1}$ are the first order perturbation of eigen-value and mode shape function, $O\left(\varepsilon^{2}\right)$ is the higher order infinitesimal of $\varepsilon$. Substituting (3) into (2), the following equations are derived if the higher order terms are ignored:

$$
E I_{0}\left(d^{4} \phi_{p}^{0} / d x^{4}+\sum_{i=1}^{m} \varepsilon_{i} d^{4} \phi_{p i}^{1} / d x^{4}\right)-\left(\lambda_{p}^{0}+\sum_{i=1}^{m} \varepsilon_{i} \lambda_{p}^{1}\right) m_{0}\left(1+\sum_{i=1}^{m} \varepsilon_{i} L \delta\left(x-x_{d i}\right)\right)\left(\phi_{p}^{0}+\sum_{i=1}^{m} \varepsilon_{i} \phi_{p i}^{1}\right)=0
$$

$$
\begin{aligned}
& \varepsilon^{0}: E I_{0} \phi_{p}^{0^{\prime \prime \prime \prime}}-m_{0} \lambda_{p}^{0} \phi_{p}^{0}=0 \\
& \varepsilon^{1}: E I_{0} \phi_{p i}^{1^{\prime \prime \prime \prime}}-m_{0} \lambda_{p}^{0} \phi_{p i}^{1}=m_{0} \lambda_{p}^{0} \phi_{p}^{0} L \delta\left(x-x_{d i}\right)+m_{0} \lambda_{p i}^{1} \phi_{p}^{0}
\end{aligned}
$$

Considering $\phi_{p i}^{1}=\sum_{q=1}^{n} \mu_{p q}^{i} \phi_{q}^{0}$, and $\phi_{p}^{1^{\prime \prime \prime \prime}}$ can be expanded as:

$$
\phi_{p i}^{1^{\prime \prime \prime \prime}}=\sum_{q=1}^{n} \mu_{p q}^{i} \phi_{q}^{0^{\prime \prime \prime \prime \prime}}=\sum_{q=1}^{n} \mu_{p q}^{i}\left(m_{0} \lambda_{q}^{0} / E I_{0}\right) \phi_{q}^{0}=\sum_{q=1}^{n} \mu_{p q}^{i} k_{q}^{4} \phi_{q}^{0}
$$

If the expression (7) is substituted into (6), the following equations are obtained:

$$
E I_{0} \sum_{q=1}^{n} \mu_{p q}^{i} k_{q}^{4} \phi_{q}^{0}-m_{0} \lambda_{p}^{0} \sum_{q=1}^{n} \mu_{p q}^{i} \phi_{q}^{0}=m_{0} \lambda_{p}^{0} \phi_{p}^{0} L \delta\left(x-x_{d i}\right)+m_{0} \lambda_{p i}^{1} \phi_{p}^{0}
$$

Considering the modal orthogonality condition, the following solutions can be obtained:

$$
\text { when } p=q, \lambda_{p i}^{1}=-m_{0} \lambda_{p}^{0} L\left[\phi_{p}^{0}\left(x_{d i}\right)\right]^{2}
$$


when $\left.\left.p \neq q, \mu_{p q}^{i}=m_{0} k_{p}^{4} L \mid \phi_{p}^{0}\left(x_{d i}\right)\right] \cdot \mid \phi_{q}^{0}\left(x_{d i}\right)\right] /\left(k_{q}^{4}-k_{p}^{4}\right)$

Considering $\int_{0}^{l} m_{d} \tilde{\phi}_{p}^{2} d x=1$, in the same way, if $p=q$ :

$$
\mu_{p q}^{i}=-1 / 2 m_{0} L\left[\phi_{p}^{0}\left(x_{d i}\right)\right]^{2}
$$

The solutions of the eigen-value and the mode shape functions can be expressed as:

$$
\begin{aligned}
& \lambda_{p}^{\%}=\lambda_{p}^{0}-m_{0} \lambda_{p}^{0} \sum_{i=1}^{m} \varepsilon_{i} L\left[\phi_{p}^{0}\left(x_{d i}\right)\right]^{2} \\
& \phi_{p}^{\%}= \begin{cases}\phi_{p}^{0}+m_{0} \sum_{i=1}^{m} \varepsilon_{i} L \sum_{q=1}^{n} k_{p}^{4} /\left(k_{q}^{4}-k_{p}^{4}\right) \cdot \phi_{q}^{0} \cdot \phi_{p}^{0}\left(x_{d i}\right) \phi_{q}^{0}\left(x_{d i}\right) & \text { when } p \neq q \\
\phi_{p}^{0}+m_{0} \sum_{i=1}^{m} \varepsilon_{i} L \sum_{q=1}^{n}(-1 / 2) \cdot \phi_{q}^{0} \cdot \phi_{p}^{0}\left(x_{d i}\right) \phi_{q}^{0}\left(x_{d i}\right) & \text { when } p=q\end{cases}
\end{aligned}
$$

\section{Case Study on a Clamped-Clamped Girder Bridge}

Case studies on the clamped-clamped girder bridge are conduced to verify the accuracy of the derivation. The parameters of the beam are: the length of the beam $L=25 \mathrm{~m}$, elastic modulus $E=2.87 \times 10^{9} \mathrm{~Pa}$, moment of inertia $I=2.9 \mathrm{~m}^{4}$, line density $m=2.303 \mathrm{t} / \mathrm{m}$. A finite element model is established as a baseline to evaluate the accuracy of the proposed method under different $\varepsilon$ and $x_{d}$. The accuracy is evaluated using $\eta$ defined below:

$$
\eta=\left(f_{P M}-f_{F E M}\right) / f_{0}
$$

where $f_{P M}$ is the modal frequency of the mass-beam system calculated using the perturbation method, $f_{F E M}$ is the modal frequency of the mass-beam system calculated using finite element method, and $f_{0}$ is the modal frequency of the un-perturbed beam.

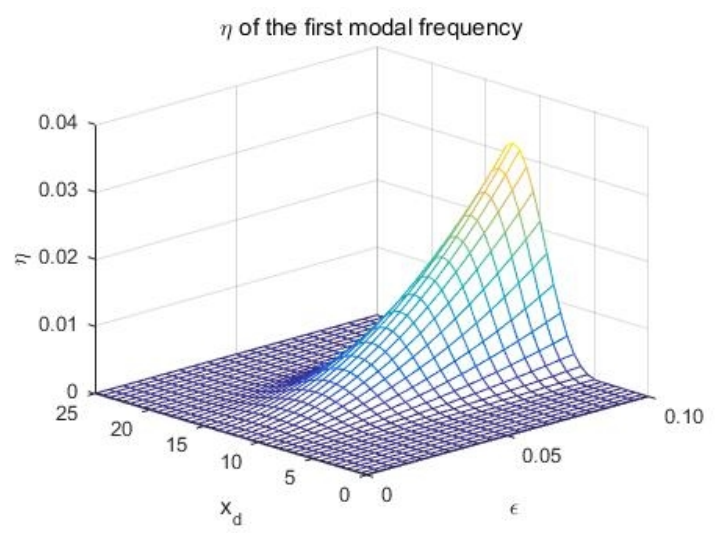

(a)

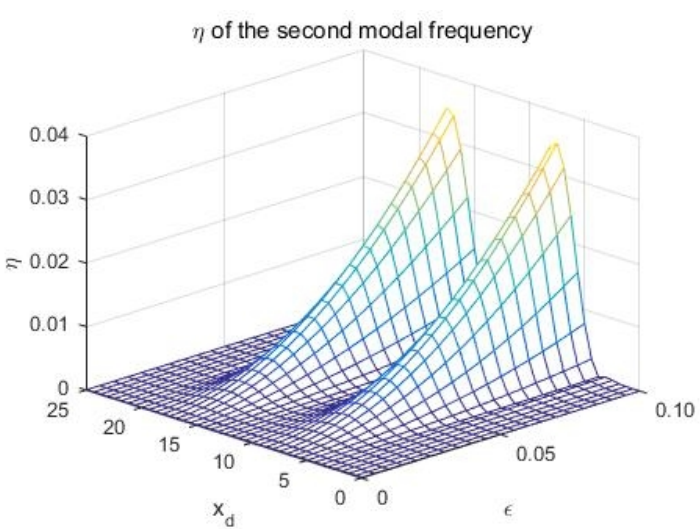

(b)

Fig. 2: The variation of $\eta$ along with $\varepsilon$ and $x_{d}$ of the first and second modal frequencies

Figure 2 shows the variation of $\eta$ along with $\varepsilon$ and $x_{d}$. The results show that the smaller the mass is, the smaller the relative error is. The relative error can be controlled within $0.5 \%$ 
when $\varepsilon \leq 0.05$ which verified that the theoretical derivation presents enough precision. Figure 3 shows the variation of $f_{P M} / f_{0}$ along with $\varepsilon$ and $x_{d}$. As shown in figure 3 , with the change of the mass location, the variation tendency of the modal frequency is the same as the square of the mode shape magnitudes. That means the maximum variation will occur when the vehicle is located at the peak; no variation will be observed if the mass is located at the inflection points.

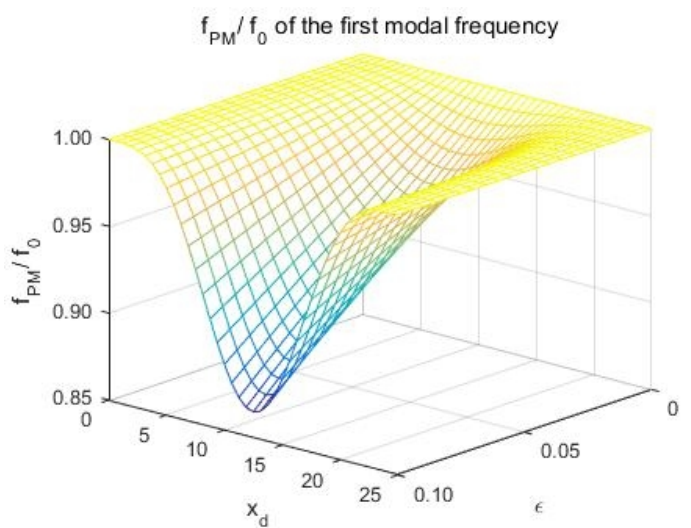

(a)

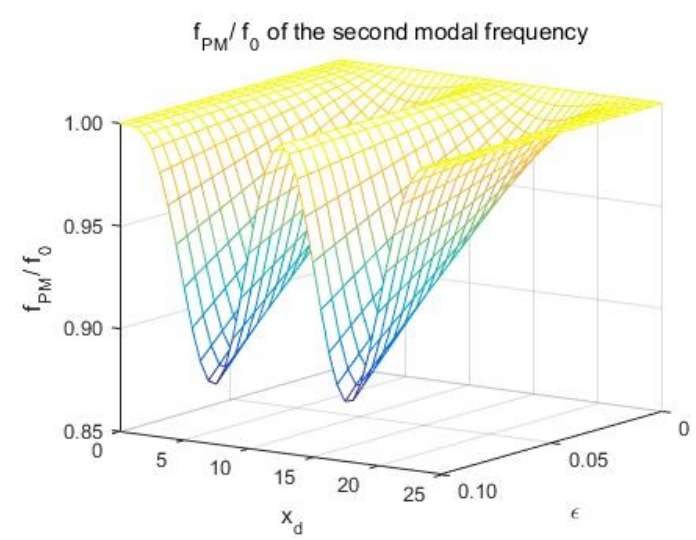

(b)

Fig. 3: The variation of $f_{P M} / f_{0}$ along with $\varepsilon$ and $x_{d}$ of the first and second modal frequencies

\section{Conclusions}

In this paper, the perturbation solution of the modal frequency and mode shape of the rigid-vehicle-load girder bridge are derived. The accuracy of this method is verified by comparing with the result of finite element method. Results of the case study on clamped-clamped girder bridge show that: Rigid mass has great influences on the bridge modal frequencies if it locates at the peak position of the mode shape; the influence on modal frequencies can be negligible if it locates at the inflection position of the mode shape.

\section{Acknowledgements}

This research was supported by 973 Program of the Ministry of Science and Technology of China (Grant No. 2013CB036305) and the SLDRCE Independent Research Fund of the Ministry of Science and Technology of China (Grant No. SLDRCE14-B-24).

\section{References}

[1] P. D. Cha: Eigenvalues of linear elastic carrying lumped masses, springs and viscous dampers [J]. Journal of Sound and Vibration, 257: 798-808. 2002.

[2] K. H. Low: Natural frequencies of a beam-mass system in transverse vibration: Rayleigh estimation versus eigenanalysis solutions [J]. International Journal of Mechanical Sciences, 45: 981-993. 2003.

[3] D. Wang: Vibration and sensitivity analysis of a beam with a lumped mass of translational and rotary inertias [J]. Journal of Vibration and Acoustics, ASME, 134: 034502-1. 2012.

[4] M. L. Lou, and Z. G. Ren: Exact solution of vibration modal characteristics of Timoshenko simply supported beam (In Chinese) [J]. Journal of Tongji University, 30(8), 2002.

[5] Z. G. Guo, and Z. Sun: Modal Analysis for Euler-Bernoulli Beam with Multiply Open Cracks Based on the Perturbation Method (In Chinese) [J]. Vibration and Shock. 2013. 